\title{
Smart And Effective Environment Monitoring Using Internet of Things
}

\author{
Sreelatha. $\mathbf{P}^{1}$, Mudassir Khan ${ }^{2}$, D. Sugumar ${ }^{3}$, Paritala Jhansi \\ Rani $^{4}$, Manikandan Ganesan ${ }^{5}$, Prashant Kumar ${ }^{6}$ \\ ${ }^{1}$ Department of Biomedical Engineering, KPR Institute of Engineering \\ and Technology, Arasur, Tamil Nadu, India. \\ ${ }^{2}$ Department of Computer Science, College of Science \& Arts \\ Tanumah, King Khalid University, Abha, Saudi Arabia. \\ ${ }^{3}$ Department of ECE, Signal Processing Lab, Karunya Institute of Technology \\ and Sciences, Karunya Nagar, Coimbatore, Tamil Nadu, India. \\ ${ }^{4}$ Department of Computer Science and Engineering, Koneru Lakshmaiah \\ Education Foundation, Vaddeswaram, Guntur, Andhra Pradesh, India. \\ ${ }^{5}$ Department of Electromechanical Engineering, Faculty of Manufacturing, \\ Institute of Technology, Hawassa University, Hawassa, Ethiopia. \\ ${ }^{6}$ Department of Chemical Engineering, Lovely Professional University, Punjab, India \\ Corresponding author email: sreelathaselvaraj@gmail.com
}

\section{ABSTRACT}

In this paper, we aim here at integrating disparate sources of information, particularly the deep neural network. The Deep Neural Network structure can intelligently track the traffic conditions in order to fulfil this task by using open data sources, whilst using sensors run by end users to provide helpful resources. We also recommended a multi-faceted architecture which permits the coexistence of different data collection methods. We have constructed a generic data structure in a relation database that gives heterogeneous data a specific interpretability. In order to make full use of the IoT potential for customers to adapt to their behaviour, data can also be offered to several suppliers. Any adjustment in our system or structures depending on the same idea would therefore encourage co-operation and convergence. It should be reminded that confidential information is generated by some users and not to be shared.

\section{KEY WORDS: IMPROVED MONITORING, ENVIRONMENT, INTERNET OF THINGS, SMART CITIES.}

\section{INTRODUCTION}

In combination with the Internet of Things (IoT), cloud inherent capabilities convert industrial automation into the process industries(Al-Turjman (2019), Boveiri (2019)).

Biosc Biotech Res Comm P-ISSN: 0974-6455 E-ISSN: 2321-4007
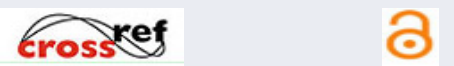

Identifiers and Pagi7) Special Issue

Received: $9^{\text {th }}$ May 2021

Pages: 258-262

Accepted after revision: $24^{\text {th }}$ July 2021

This is an open access article under Creative

Commons License Attribn 4.0 Intl (CC-BY).

DOI: $h t t p: / / d x . d o i . o r g / 10.21786 / b b r c / 14.7 .57$
The IoT services compliment emerging computing and open networking on cloud capabilities. With cloud benefits, several more services can increase automation in smart manufacturing businesses.

Development and design necessitate increased cooperation within the entire company and industry spectrum. The large number of manufacturing objects will centrally be controlled by collaborative manufacturing models or processes, for example the cloud-based resource/object management system (Ma et. al. (2019)). IMs are the foundation of every firm who aims to use modern technology in the development of value-adding processes and services within the framework of Industry 4.0. (Basu et. al. (2018)). 
Recently, IoT integration with the cloud demands adequate conversion and the transition is not achieved by engineering principles. Due to its dependability, performance and security, the implementation of cloud-integration in IoT components is strictly limited (Xu (2019), AbdelBasset (2020), Zhang (2018), Cai (2020), Ejaz (2020)). Therefore, the IoT devices must be deployed together to carry out the necessary duty that can modify the standard automation model efficiently in the industries. Recently, many approaches of deep learning (Nguyen (2019), Abdelmoneem (2020), Rahbari (2019), Shanthan (2017), Fan (2017), Lakhan (2021)) are used, particularly deep neural networks (CNNs), for cloud and IoT automatic industry systems. In addition, there are a number of modelbased engineering methods (Ismail (2018), Chang (2021), Saravanan (2016), Saravanan (2016), Sumathi A (2015)) which contribute to cloud-based job optimization.

In this research, a model-based engineering analysis is offered which executes industrial workflows in cloudbased IoT. The built-in IoT on the cloud combines Cloud functionality with open IoT connections. During this investigation, significant energy is used during the validation phases to track the reference signal and the pump requires maximum voltage. We apply the ML method, the Convolutional Neural Network (CNN) to optimise the workingflow operation to improve the tracking of the reference signal with less energy and minimum voltage to the pump. The optimised operation of this integrated workflow resolves the optimization process repeatedly to achieve the required output.

Background: In today's changing world, fast and accurate task handling is vital. A cognitive or intelligent model of task programing algorithms that can deal with heterogeneous multi-processor environments like cloud computing is important to ensure that tasks are assigned to the right processor on a specific timetable. The topic of planning a group of IoT application tasks in a cloud environment with multi-processors (IOT) has been addressed in this work. The aim of any work scheduling method is to plan processors' tasks so as to reduce the overall performance without infringing on the above limitations. The Scheduling of Multiprocessors is considered to be an NP-hard issue (Al-Turjman et. al. (2019)). IoT tasks are shown in the form of a run time matrix and their associated time needed to run on a particular processor (ETM). Different IoT tasks on different processors are supposed to have various execution times (Boveiri (2019), Ma (2019).

The preceding task relation is represented as a directed acyclic graph, with vertices representing calculation time (the job is assigned to a processor) and guided edges reflecting the time of communication between these vertices. Only if two operations are planned on two distinct processors will communication time be taken into account. Recent development in IoT applications makes it difficult to plan different jobs in the cloud, such as multiprocessor environments, which are interdependent. Given the heterogeneity in type of application duties in the IoT market, it is crucial that IoT apps are planned and loaded efficiently (Ismail (2018), Chang (2021), Saravanan (2016),
Saravanan (2016), Sumathi (2015)). Task scheduling is often approached utilising heuristic techniques which, while taking into account the imposed limitations, offer constructive and optimal solutions in polynomial time.

It looks for a road in the space of the solution to ignore some feasible paths (Saravanan V (2012), Sobhanayak (2020)). Task queues and lists are used to allocate a priority value for every task based on certain criteria in the task scheduling category. The solution space is narrower and such algorithms are particularly efficient. Nevertheless, these algorithms rely heuristically very much on them. The task-planning algorithm category clusters maximise the number of processors so that their performance is minimised. The combinatory for the solution space is used by the guided random search-based algorithms. Such algorithms demand a large number of candidates in search area 65 , yet they have proven quite effective in a range of scheduling issues.

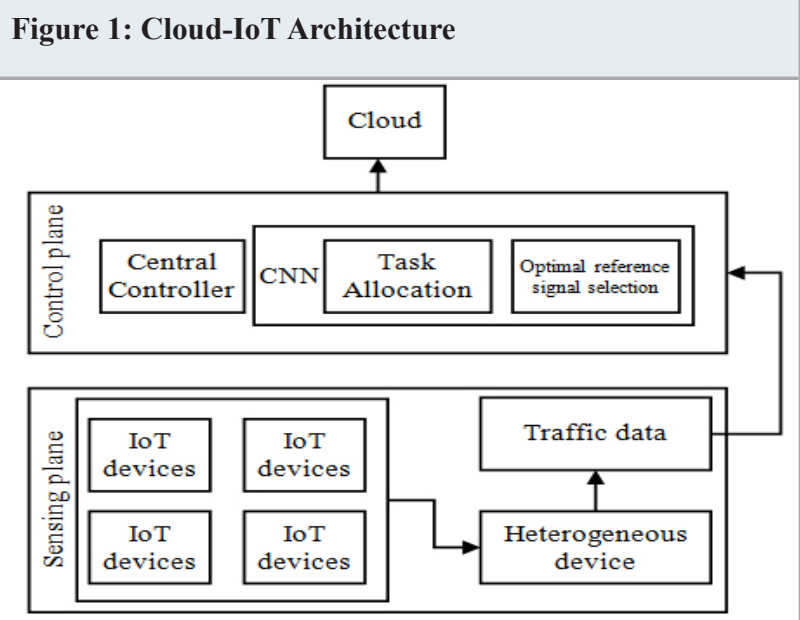

Figure 2: CNN Architecture for proposed Cloud-IoT model

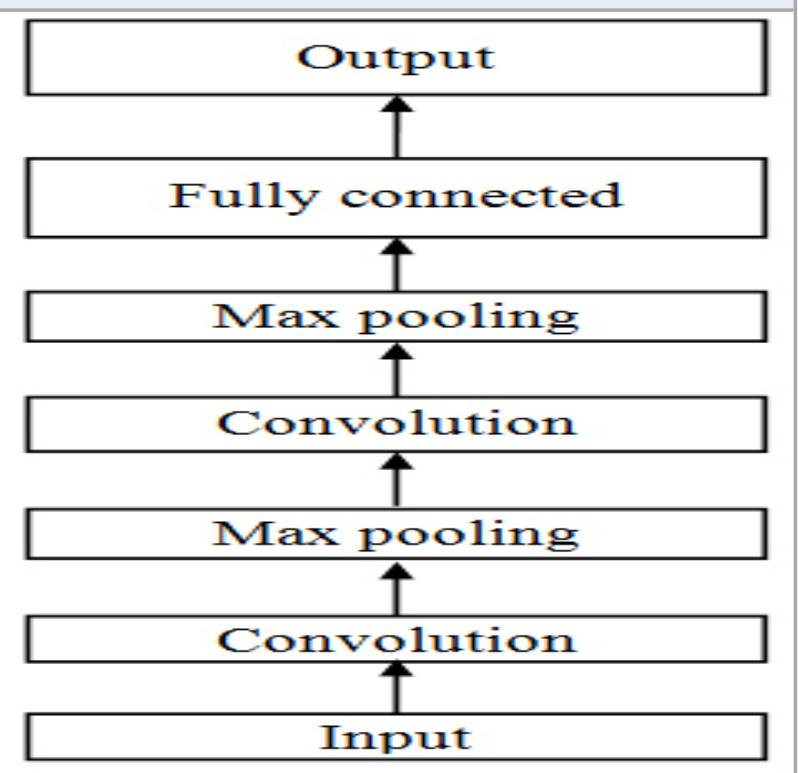


Proposed Method: In this section we provide an architecture of the cloud-based IoT industrial workflow which integrates the suggested DBB-MBE to better choose the reference signal for optimised process flow. This allows optimal maintenance of the pumping action at maximum voltage. In (Nguyen et. al. (2019)), where the present study discovers the gap in the previous model and resolves optimally the CNN task that identifies the greatest voltage for pumping operations, the selection of the reference voltage is not concentrated. Further, the study failed to provide information on the schedule of tasks, where the current model CNN collects and plans the quick purchase of IoT device input signals according to the availability of VMs for the cloud environment. Figure 1 shows the architecture of the proposed CNN-based Cloud-IoT platform.

Model Based Engineering: The method MBE is deemed appropriate in the increase of the abstraction level and in the automation of fault-prone and labor-intensive tasks. This reduces design costs and enhances data sharing, reusability and model verification. Because the aforementioned factors are increasingly automated from the cloud, the automation model tends to become complex with interactions of various heterogeneous entities and domains. This paper examines the multi-view modelling of the industrial automation system, in which MBE overcomes the challenge of optimising IoT solutions in the cloud.

3.2. Prediction using CNN: The model-based design is done utilising a workflow that helps to implement the model predictive controller when using the cloud-IoT-based process control apps. In the model-based design workflow, the need includes the specified objective function for tracking the reference signal, optimally a minimum level of energy linked to the maximum-voltage pumping limits, as specified for control design needs. A goal to optimise modelbased design is selected to provide the objective function of minimum voltage $(\min (\mathrm{V})$ for pumping operations with the maximum voltage constraint maximum $(\mathrm{V})$. The controller and the procedure employing CNN are here performed for control design checking in the virtual environment (given in Figure 1).

Figure 2 shows the architecture of CNN. It comprises of a string of convolutional and max levels of pooling, and only their previous layer gives each layer connections. The input data rate of the IoT devices is monitored with the VM resource signal and the reference signal voltage based on the signal restriction is an overall, hierarchic feature extractor. The classification takes place with the layers completely linked. The joint optimization of all configurable parameters reduces the error in the misclassification of the training set.

The time for the job execution J(i) is defined in terms of a non-negative variables $(\mathrm{n} \times \mathrm{k})$ in the interval $\mathrm{I}(\mathrm{j})$,

$x(i, j)$, where $1 \leq i \leq n ; 1 \leq j \leq k$.

Non-negative variable is expressed as below: $x(i, j) \geq 0, \forall i, j$, where $1 \leq i \leq n, 1 \leq j \leq k$

The scheduling constraints are defined to increase the VMs capacity over an interval kas below:

$\left(\sum_{i=1}^{n} x(i, j)\right) \leq t(j+1)-t(j), \forall j, 1 \leq j \leq k$

The CNN on resolves the problem of scheduling using following expression:

$\varepsilon \frac{d \mathrm{x}}{d t}=C^{T} g_{[,, h]}\left(C_{\mathrm{x}-f}\right)-\sigma A^{T} g_{[0,1]}(A \mathrm{x}-b)$

The problem of finding the optimum solution under overload is seen as an NP difficulty, as it makes the necessary scheduling more complex. The presence of the utility function and the efficiency of the work plan declines linearly after its expiry date. In such situations, the cumulative maximisation of lateness increases the usefulness to the maximum level.

Figure 3: Computational Time of task scheduling during (a) training state and (b) testing state

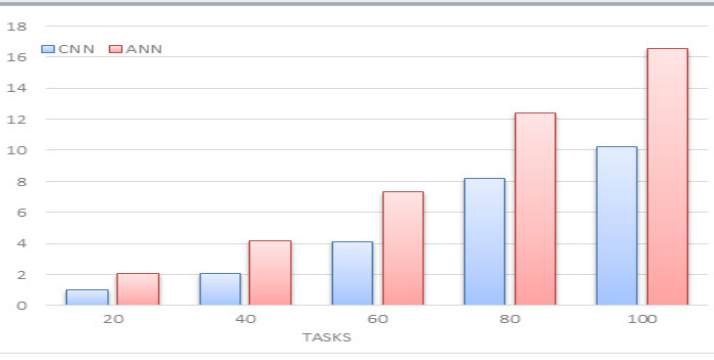

(a)

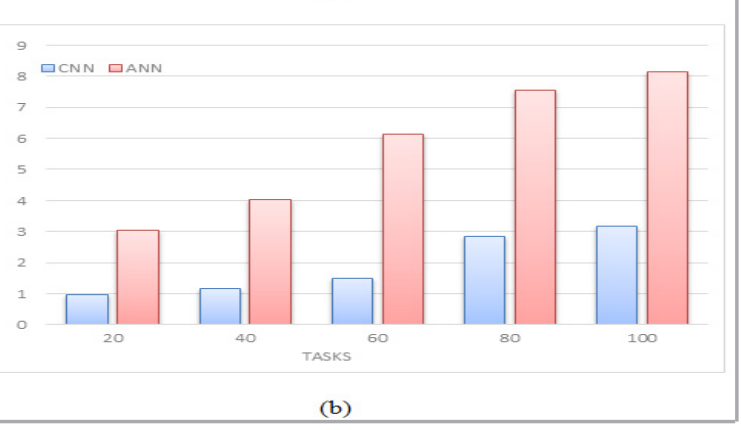

RESULTS AND DISCUSSION

The validations are based on the iterative procedure and design changes can be confirmed using the acquired findings. The simulation checks the benefits of MBE integration with Cloud-IoT. The method proposed is compared to the benchmark method used to measure the effectiveness of the machine learning method proposed. It analyses further whether the referential signal has been tracked with lowered timing and voltage in order to pump. Finally, the study analyses cloud-IoT modelling as an additional component of the study based on assigned resources of data collection and acquisition in Cloud and 
IoT resources and the operation of the MBE-CNN in IoT input processing for the decision of the optimal reference signal in the cloud.

Figure 3 illustrates the computer time results before and after the reference signal voltage has been optimised by means of CNN. The results demonstrate that effective reference signal selection has enhanced the way the complete task can be optimised and therefore the time to calculate the entire workflow has decreased successfully compared with the prior model (Nguyen et. al. (2019)) based engineering.

Figure 4: Cost of task schedulingduring (a) training state and (b) testing state

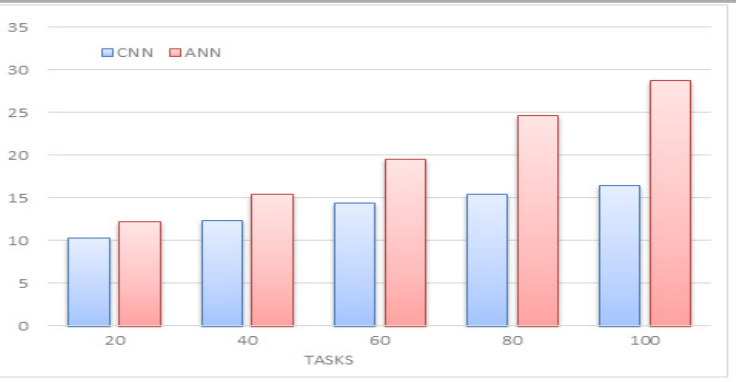

(a)

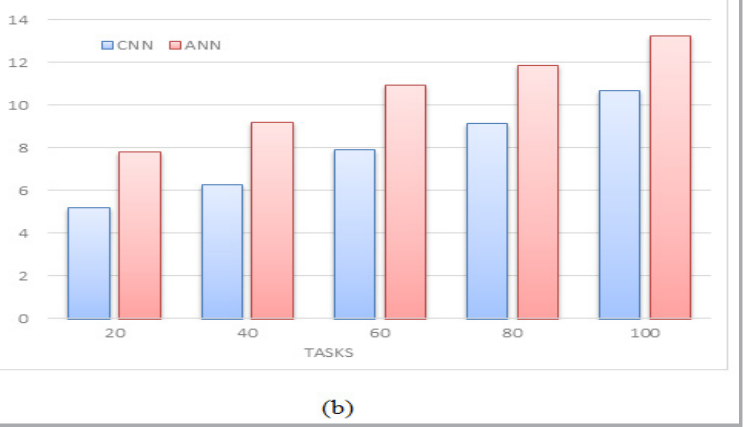

Figure 5: Task response time

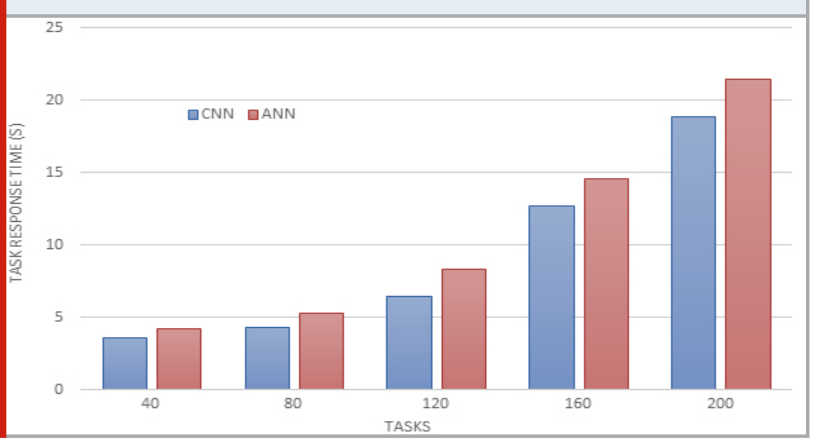

Figure 4 illustrates the costs $(\$)$ for the planned job before and after optimization with the help of CNN, which successfully includes the optimisation of the IoT system for industrial automation cloud jobs with improved pumping voltage selection in the background. Figure 4 displays the cost and Figure 5 display the task response time. This optimum choice allowed the system to lower its operational cost compared to the current framework, i.e. the method in (Nguyen et. al. (2019)).

\section{CONCLUSION}

This paper presents a model based engineering that uses the cloud-based IoT industrial workflow by merging $\mathrm{G}$ cloud with open IoT connection. The reference signal tracking is optimised so that energy is reduced and the pumping voltage is selected as optimally as possible during the testing stage. The CNN optimises efficiently the functioning of the workflow to optimise the pumping voltage and reduce energy consumption. With its repeated repetition, CNN solves the workflow operation optimally. The simulation results against the benchmark approach validate this, as the suggested method resolves energy usage optimally through optimal pumping voltage.

\section{REFERENCES}

Abdel-Basset, M., Mohamed, R., Elhoseny, M., Bashir, A.K., Jolfaei, A. and Kumar, N., 2020. Energy-aware marine predators algorithm for task scheduling in IoTbased fog computing applications. IEEE Transactions on Industrial Informatics, 17(7), pp.5068-5076.

Abdelmoneem, R.M., Benslimane, A. and Shaaban, E., 2020. Mobility-aware task scheduling in cloud-Fog IoTbased healthcare architectures. Computer Networks, 179, p. 107348 .

Al-Turjman, F., Hasan, M.Z. and Al-Rizzo, H., 2019. Task scheduling in cloud-based survivability applications using swarm optimization in IoT. Transactions on Emerging Telecommunications Technologies, 30(8), p.e3539.

Boveiri, H.R., Khayami, R., Elhoseny, M. and Gunasekaran, M., 2019. An efficient Swarm-Intelligence approach for task scheduling in cloud-based internet of things applications. Journal of Ambient Intelligence and Humanized Computing, 10(9), pp.3469-3479.

Basu, S., Karuppiah, M., Selvakumar, K., Li, K.C., Islam, S.H., Hassan, M.M. and Bhuiyan, M.Z.A., 2018. An intelligent/cognitive model of task scheduling for IoT applications in cloud computing environment. Future Generation Computer Systems, 88, pp.254-261.

Cai, X., Geng, S., Wu, D., Cai, J. and Chen, J., 2020. A Multicloud-Model-Based Many-Objective Intelligent Algorithm for Efficient Task Scheduling in Internet of Things. IEEE Internet of Things Journal, 8(12), pp.96459653.

Ejaz, W., Ahmed, A., Mushtaq, A. and Ibnkahla, M., 2020. Energy-efficient task scheduling and physiological assessment in disaster management using UAV-assisted networks. Computer Communications, 155, pp.150-157. Ezhilarasi, G.D., Latchoumi, T.P. and Balamurugan, 
K., 2020. UIP_A Smart Web Application to Manage Network Environments, Advances in Intelligent systems and computing book series.

Fan, J., Wei, X., Wang, T., Lan, T. and Subramaniam, S., 2017, December. Deadline-aware task scheduling in a tiered IoT infrastructure. In GLOBECOM 2017-2017 IEEE Global Communications Conference (pp. 1-7). IEEE.

Ismail, L. and Materwala, H., 2018. Energy-aware vm placement and task scheduling in cloud-iot computing: Classification and performance evaluation. IEEE Internet of Things Journal, 5(6), pp.5166-5176.

Lakhan, A., Mastoi, Q.U.A., Elhoseny, M., Memon, M.S. and Mohammed, M.A., 2021. Deep neural network-based application partitioning and scheduling for hospitals and medical enterprises using IoT assisted mobile fog cloud. Enterprise Information Systems, pp.1-23.

Ma, X., Gao, H., Xu, H. and Bian, M., 2019. An IoTbased task scheduling optimization scheme considering the deadline and cost-aware scientific workflow for cloud computing. EURASIP Journal on Wireless Communications and Networking, 2019(1), pp.1-19.

Mishra, P., Jimmy, L., Ogunmola, G.A., Phu, T.V., Jayanthiladevi, A. and Latchoumi, T.P., 2020, December. Hydroponics cultivation using real time iot measurement system. In Journal of Physics: Conference Series (Vol. 1712, No. 1, p. 012040). IOP Publishing.

Nguyen, B.M., Thi Thanh Binh, H. and Do Son, B., 2019.

Evolutionary algorithms to optimize task scheduling problem for the IoT based bag-of-tasks application in cloud-fog computing environment. Applied Sciences, 9(9), p.1730.

Prasath, S. (2020). Probabilistic Mceliece Public-Key Cryptography Based Identity Authentication For Secured Communication In Vanet. Solid State Technology, 63(6), 10167-10182.

Prasath, S. Validating Data Integrity in Steganographed Images using Embedded Checksum Technique. International Journal of Computer Applications, 975, 8887.

Rahbari, D. and Nickray, M., 2019. Low-latency and energy-efficient scheduling in fog-based IoT applications. Turkish Journal of Electrical Engineering \& Computer Sciences, 27(2), pp.1406-1427.

Saravanan, V. and Raj, V.M., 2016. A Seamless Mobile Learning and Tension Free Lifestyle by QoS Oriented Mobile Handoff. Asian Journal of Research in Social Sciences and Humanities, 6(7), pp.374-389.
Saravanan, V. and Raj, V.M., 2016. Maximizing QoS by cooperative vertical and horizontal handoff for tightly coupled WiMAX/WLAN overlay networks. Cluster Computing, 19(3), pp.1619-1633.

Saravanan, V. and Sumathi, A., 2012. Handoff mobiles with low latency in heterogeneous networks for seamless mobility: A survey and future directions. European Journal of Scientific Research, 81(3), pp.417-424.

Shanthan, B.J., Kumar, A.D.V., Govindrajan, E. and Arockian, L., 2017. Scheduling for internet of things applications on cloud: A review. Imperial Journal of Interdisciplinary Research, 3(1), pp.1649-1653.

Shukla, A., Kalnoor, G., Kumar, A., Yuvaraj, N., Manikandan, R. and Ramkumar, M., 2021. Improved recognition rate of different material category using convolutional neural networks. Materials Today: Proceedings.

Sobhanayak, S., Jaiswal, K., Turuk, A.K., Sahoo, B., Mohanta, B.K. and Jena, D., 2020. Container-based task scheduling for edge computing in IoT-cloud environment using improved HBF optimisation algorithm. International Journal of Embedded Systems, 13(1), pp.85-100.

Sumathi, A. and Saravanan, V., 2015. Bandwidth based vertical handoff for tightly coupled wimax/wlan overlay networks.

Sumathy, S., Revathy, M. and Manikandan, R., 2021. Improving the state of materials in cybersecurity attack detection in 5G wireless systems using machine learning. Materials Today: Proceedings.

Venkata Pavan, M., Karnan, B. and Latchoumi, T.P., 2021. PLA-Cu reinforced composite filament: Preparation and flexural property printed at different machining conditions. Advanced Composite Materials, https://doi. org/10.1080/09243046.2021, 1918608.

Xu, J., Hao, Z., Zhang, R. and Sun, X., 2019. A method based on the combination of laxity and ant colony system for cloud-fog task scheduling. IEEE Access, 7, pp.116218116226.

Yuvaraj, N., Chang, V., Gobinathan, B., Pinagapani, A., Kannan, S., Dhiman, G. and Rajan, A.R., 2021. Automatic detection of cyberbullying using multi-feature based artificial intelligence with deep decision tree classification. Computers \& Electrical Engineering, 92, p.107186.

Zhang, G., Shen, F., Zhang, Y., Yang, R., Yang, Y. and Jorswieck, E.A., 2018, October. Delay minimized task scheduling in fog-enabled IoT networks. In 2018 10th International Conference on Wireless Communications and Signal Processing (WCSP) (pp. 1-6). IEEE. 